Document downloaded from:

http://hdl.handle.net/10251/60063

This paper must be cited as:

Monsoriu Serra, JA.; Gimenez Valentin, MH. (2011). Undergraduate experiment with fractal diffraction gratings. European Journal of Physics. 32:687-694. doi:10.1088/01430807/32/3/005.

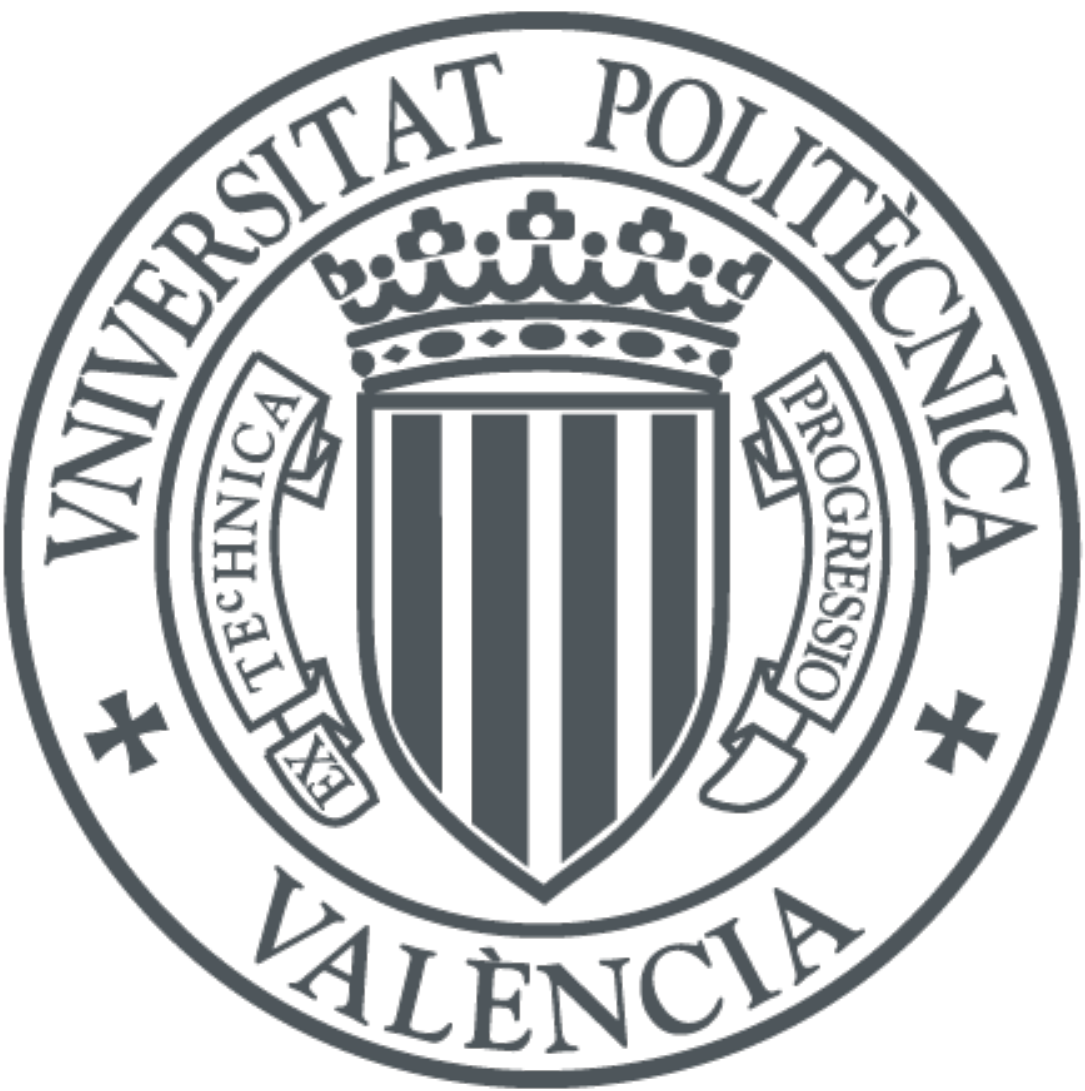

The final publication is available at

http://dx.doi.org/10.1088/0143-0807/32/3/005

Copyright European Physical Society

Additional Information 


\title{
Undergraduate experiment with fractal diffraction gratings
}

\author{
Juan A. Monsoriu ${ }^{1, *}$, Walter D. Furlan², Amparo Pons², \\ Juan C. Barreiro ${ }^{2}$, and Marcos H. Giménez ${ }^{3}$
}

${ }^{1}$ Centro de Tecnologías Físicas, Universidad Politécnica de Valencia, E-46022 Valencia, Spain

${ }^{2}$ Departamento de Óptica, Universidad de Valencia, E-46100 Burjassot (Valencia), Spain

${ }^{3}$ Instituto de Matemática Multidisciplinar, Universidad de Valencia, E-46100 Burjassot (Valencia), Spain

*Corresponding author: jmonsori@fis.upv.es

\begin{abstract}
We present a simple diffraction experiment with fractal gratings based on the Triadic Cantor Set. Diffraction by fractals is proposed as a motivating strategy for the students of optics about the potential applications of optical processing. The Fraunhofer diffraction patterns were obtained with the standard equipment present in most undergraduate physics labs and compared with that obtained with conventional periodic gratings. It is shown that fractal gratings produce self-similar diffraction patterns which can be evaluated analytically. A very good accuracy is obtained between experimental and numerical results.
\end{abstract}

Keywords: Fractal; Diffraction; Cantor set

PACS Numbers: $42.25 . \mathrm{Fx}, 42.30 . \mathrm{Kq}$ 


\section{Introduction.}

When diffraction optics is introduced in the first courses of physics typically the considered structures are as single or double slits and periodic gratings. These are classical examples of objects used to teach the diffraction phenomena but it is rarely used to show the potential application of diffraction in signal processing. In our opinion nonconventional diffractive objects as fractals could be used to this purpose.

Fractal geometry is exceptionally fruitful and has been also identified in many other scientific areas such as biology, medicine, electronics, geomorphology, and even stocks markets. Mathematically, the concept of fractal is associated with a geometrical object which i) is self-similar (i.e., the object is exactly or approximately similar to a part of itself) and, ii) has a fractional (or noninteger) dimension [1]. Fractals are obtained by performing a basic dividing operation, called generator, on a given geometrical object called initiator. By repeating sequentially this process on each one of the parts resulting from the division, a multiple levels object, composed of sub-units of itself is created that resembles the structure of the whole object [2].

In optics, fractal structures, ranging from simple one-dimensional (1D) systems $[3,4]$ to complex $2 \mathrm{D}$ objects $[5,6]$ have been extensively studied. Specifically, diffraction gratings based on the Fractal Cantor Set sequences have been analyzed [7,8]. The fractal profile of these structures leads to a Fraunhofer diffraction patterns with selfsimilar properties, i.e., the variation of the Fourier spectrum with the spatial frequency at each higher stage is a modulated version of that associated with the previous step with an appropriate scaling of the frequency range. As a consequence of this fractal property, these structures exhibit a certain number of subsidiary diffraction peaks around the main peaks. This self-similar property has been also obtained with other dif- 
fractive optical elements, as for example, with different kind of fractal diffractive lenses $[9,10]$.

In this paper we present a simple experiment to verify the self-similar properties of Fraunhofer diffraction patterns produced by fractal gratings (FGs) with the standard equipment present in most undergraduate physics laboratories. For comparison, the Fraunhofer patterns of regular periodic gratings (PGs) are also obtained. From a didactic point of view, the developed experiment is very stimulating because it also becomes a way for introducing students into basic research activities.

\section{Fractal gratings based on the Triadic Cantor set.}

One of the simplest fractals is the Triadic Cantor set, shown in Fig. 1(a), which can be obtained by means of an iterative construction. The first step $(S=0)$ is to define a segment of unit length. The next one $(S=1)$ is to divide the segment in three equal parts of length $1 / 3$ and removing the central one. The ternary set created by repeating this process ad infinitum on the remaining segments.

(a) Fractal structure

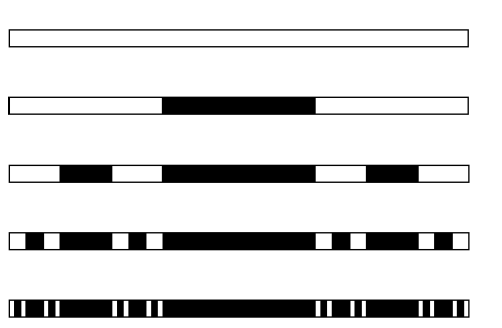

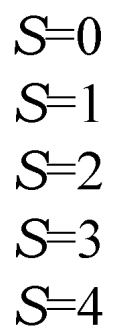

(b) Periodic structure

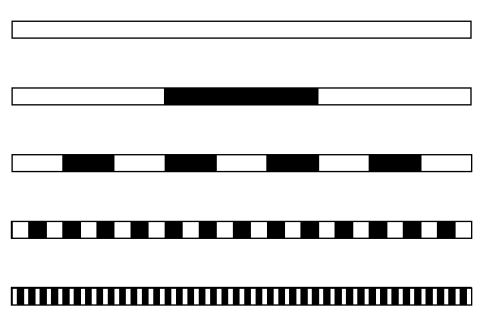

Figure 1. (a) Triadic Cantor Set and (b) the equivalent periodic structure at different stages of growth $S$. 
The triadic Cantor set contains all points in the interval $[0,1]$ that are not deleted at any step in this infinite process. In general, at the stage $S$, there are $2^{S}$ segments of length $3^{-S}$ with $2^{S}-1$ gaps in between. In Fig. 1(a), only the four first stages are shown for clarity. Note that the $S$-th stage Cantor set can be interpreted as a quasiperiodic distribution of segments which can be obtained by removing some segments in a finite periodic distribution as shown in Fig. 1(b). This distribution at stage $S$ has $\left(3^{S}+1\right) / 2$ segments of length $3^{-S}$, separated by gaps of the same length, so the period of this finite structure is $\Lambda=2 \cdot 3^{-S}$.

Based on the above scheme we propose FGs composed of slits distributed according to the Triadic Cantor Set, as it is shown in Fig. 2. In order to show the selfsimilarity properties of the diffracted field for different values of $S$ we have scaled the whole structure leaving in each stage the slits with the same width $a$. In this way the spacing between the diffraction orders will be preserved. Therefore, in our objects the grating length is given by $L=a 3^{S}$ and the period of the equivalent periodic grating is $\Lambda$ $=2 a$ (see Fig. 2). In mathematical terms, a Cantor grating of order $S$ can be represented by a binary function transmittance $t_{\mathrm{F}}(x, y)$ given by

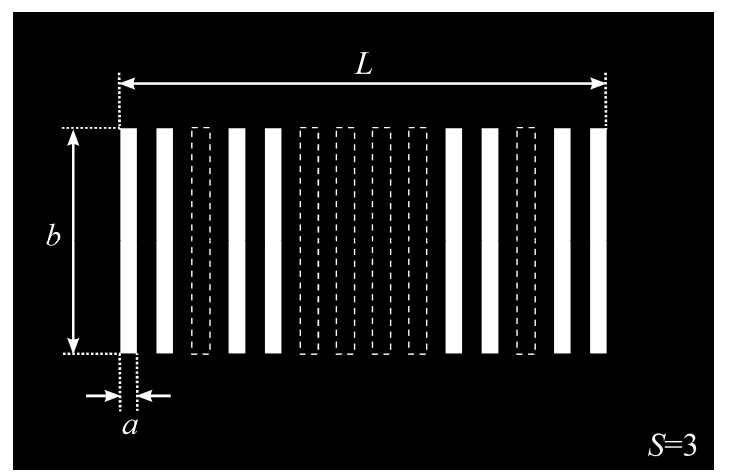

Figure 2. Fractal grating based on the Triadic Cantor Set shown in Fig. 1 at stage of growth $S=3$. The dashed lines define de complimentary slits of the equivalent periodic grating. 


$$
t_{\mathrm{F}}(x, y)=\prod_{i=0}^{S} \operatorname{rect}\left(\frac{\operatorname{Mod}\left[x-\frac{L}{2}+3^{i} a, 2 \cdot 3^{i} a\right]}{2 \cdot 3^{i} a}\right) \operatorname{rect}\left(\frac{x}{L}\right) \operatorname{rect}\left(\frac{y}{b}\right),
$$

where $\operatorname{rect}(x)=1$ for $|x|<1 / 2$ and 0 otherwise. In this equation $\operatorname{Mod}(x, y)$ gives the remainder on division of $x$ by $y$. The transmittance of the equivalent PG (represented in Fig. 2 by the dashed lines slits plus the FG) is given by

$$
t_{\mathrm{P}}(x, y)=\operatorname{rect}\left(\frac{\operatorname{Mod}\left[x-\frac{L}{2}+a, 2 a\right]}{2 a}\right) \operatorname{rect}\left(\frac{x}{L}\right) \operatorname{rect}\left(\frac{y}{b}\right) .
$$

\section{Theoretical Fraunhofer diffraction patterns.}

Let us consider a diffraction grating with transmittance $t(x, y)$, illuminated by a monochromatic plane wave (with wavelength $\lambda$ ). The Fraunhofer diffraction pattern is generated at the back focal plane of a lens placed against the grating. Within the scalar approximation, the focal irradiance distribution is given by the Fourier transform of the transmittance function [12],

$$
I(x, y)=\left(\frac{A}{\lambda f}\right)^{2}\left|\int_{-\infty}^{\infty} t\left(x_{o}, y_{o}\right) \exp \left(-\mathrm{i} \frac{2 \pi}{\lambda f}\left(x \cdot x_{o}+y \cdot y_{o}\right)\right) d x_{o} d y_{o}\right|^{2}
$$

where $f$ is the focal length of the lens and $\mathrm{A}$ is the amplitude of the incident plane wave.

To compare the diffraction properties of a FG with its associated PG, we will obtain analytically the Fraunhofer diffraction irradiance distributions by replacing in the above equation the transmittance function $t(x, y)$ by Eqs. (1) and (2), respectively. 
For the first case, by using the dimensionless transversal coordinates $u=\frac{a}{\lambda f} x$ and $v=\frac{b}{\lambda f} y$, and using of the convolution theorem for the Fourier transform, Eq. (3) can be rewritten as

$$
I_{\mathrm{F}}(u, v)=\frac{1}{4^{S}} \prod_{i=1}^{S} \frac{\operatorname{Sin}^{2}\left(4 \pi u 3^{S-i}\right)}{\operatorname{Sin}^{2}\left(2 \pi u 3^{S-i}\right)} \operatorname{Sinc}^{2}(u) \operatorname{Sinc}^{2}(v) .
$$

For the associated PG, Eq. (3) leads to the well-known result [12]:

$$
I_{\mathrm{P}}(u, v)=\frac{1}{M^{2}} \frac{\operatorname{Sin}^{2}(M 2 \pi u)}{\operatorname{Sin}^{2}(2 \pi u)} \operatorname{Sinc}^{2}(u) \operatorname{Sinc}^{2}(v) .
$$

In Eq. (5) $M$ is the number of transparent slits and is given by $\left\lceil\frac{3^{S}}{2}\right\rceil$, where $\lceil x\rceil$, called the ceiling of $x$, denotes the smallest integer greater than or equal to $x$. Note that Eqs. (4) and (5) have been normalized to $I(0,0)=1$.

Profiles of the Fraunhofer irradiance produced by FGs with different stages of growth $S$, are shown in Fig. 3(a). The irradiance of the associated PGs is shown in the same figure for comparison. It can be seen that the main diffraction peaks in both cases coincide. However, the irradiance for the FG exhibits a characteristic fractal profile. Note also that the irradiance for the FG at each higher stage is a modulated version of that associated with the previous stage. That is, the Fraunhofer diffraction pattern reproduces the self-similarity of the grating distribution. 

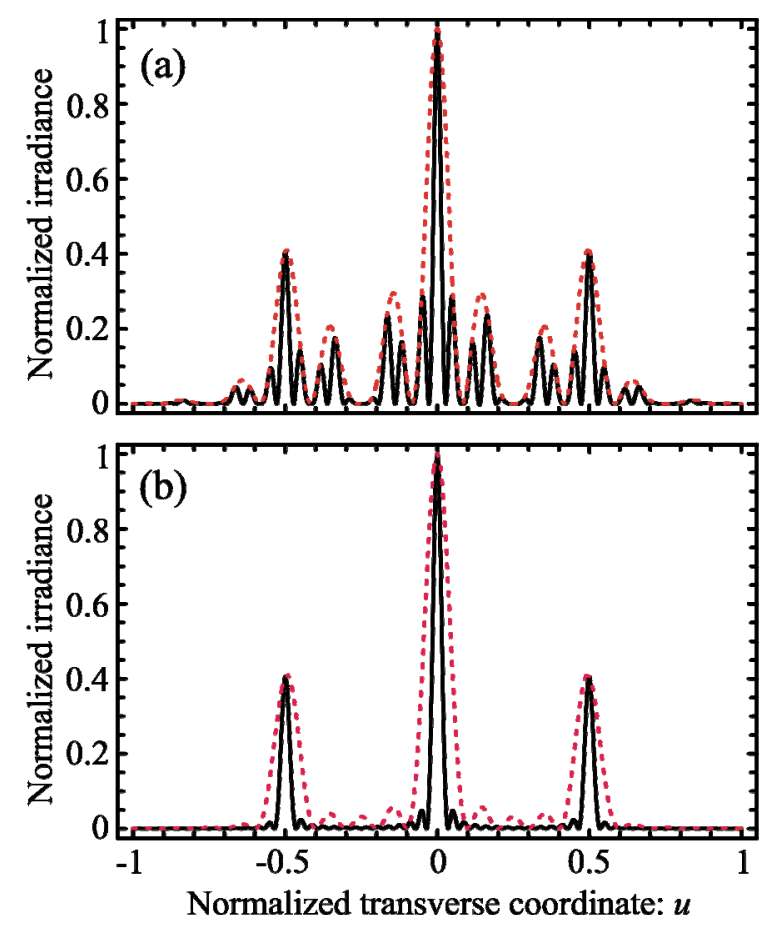

Figure 3. (a) Normalized Fraunhofer irradiance vs. the transverse coordinate $u$ for a FGs $(S=2$; dashed curve and $S=3$; solid curve) and (b) for its associated PG. In both cases $v=0$.

\section{Experimental results.}

The setup employed to obtain experimentally the Fraunhofer diffraction patterns is shown in Fig. 4. A He-Ne laser $(\lambda=632.8 \mathrm{~nm})$ connected to optical fiber is used as a point-source illuminator (A). A lens of focal distance $f=200 \mathrm{~mm}$ (B) collimates the beam. The diffraction gratings $(\mathrm{C})$ were printed and then photographically reduced onto $35 \mathrm{~mm}$ slides. The sizes of the resulting slits are $a=0.18 \mathrm{~mm}$ and $b=5.39 \mathrm{~mm}$. The Fraunhofer diffraction patterns are obtained at the focal plane of the lens (D) $(f=400 \mathrm{~mm})$. A microscope objective $(4 \mathrm{x})$ forms an image of these diffraction patterns onto a CCD detector $(\mathrm{E})$. The diffraction patterns can be visualized directly in the monitor $(\mathrm{F})$ and using an image acquisition software the patterns are saved in the computer (G) as JPEG files (256-level gray scale and 764x576 pixels). 


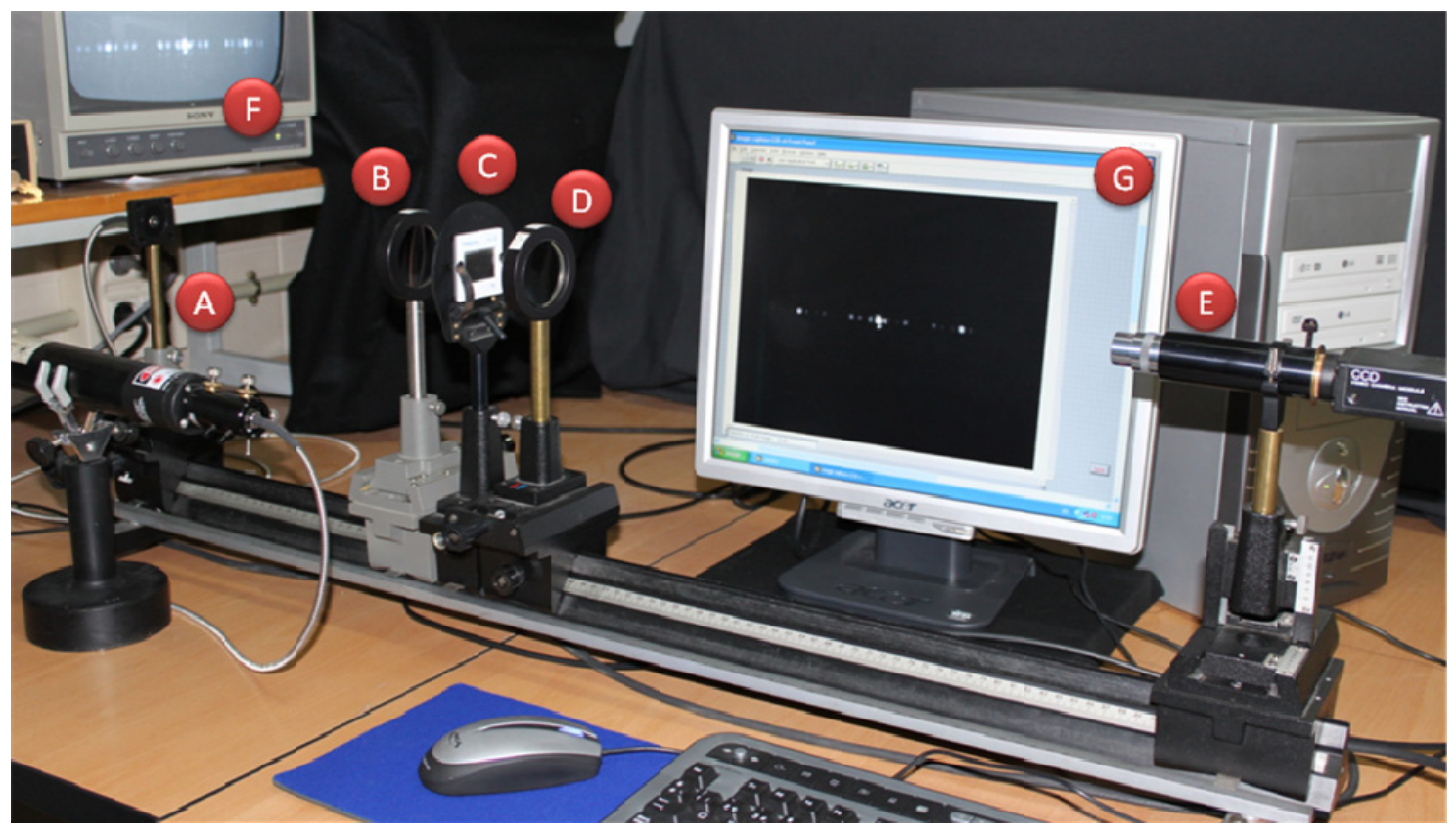

Figure 4. Experimental setup employed to obtain experimentally the Fraunhofer diffraction patterns.

Figure 5 shows the experimental diffraction patterns obtained with FGs of orders $S=2$ and $S=3$ and the corresponding patterns obtained with the equivalent periodic structures. Although the patterns are recorded as images of $764 \times 576$ pixels, only the central regions of interest are shown in this figure. Note that the zero-order diffraction peaks are saturated in order to detect the high order diffraction peaks. From Fig. 5 it is clear the self-similar behavior of FGs. In fact, any wide peak at stage $S=2$ is transformed into three narrower peaks at stage $S=3$ and nine peaks at stage $S=4$ (not shown in Fig. 5). The image files are transformed with Mathematica in gray-level irradiance matrices, $I_{\exp }(i, j)$, with $i=1,2, \ldots 764, j=1,2 \ldots 576$, and $0 \leq I_{\exp } \leq 255$. Taking into account the symmetry properties of the diffraction patterns and using the Mathematica's function $\operatorname{Mean}\left[\operatorname{Position}\left[I_{\mathrm{exp}}, \operatorname{Max}\left[I_{\mathrm{exp}}\right]\right]\right]$ it is easy to locate the central pixel, $\left(i_{0}, j_{0}\right)$, of the zeroorder diffraction peak. 

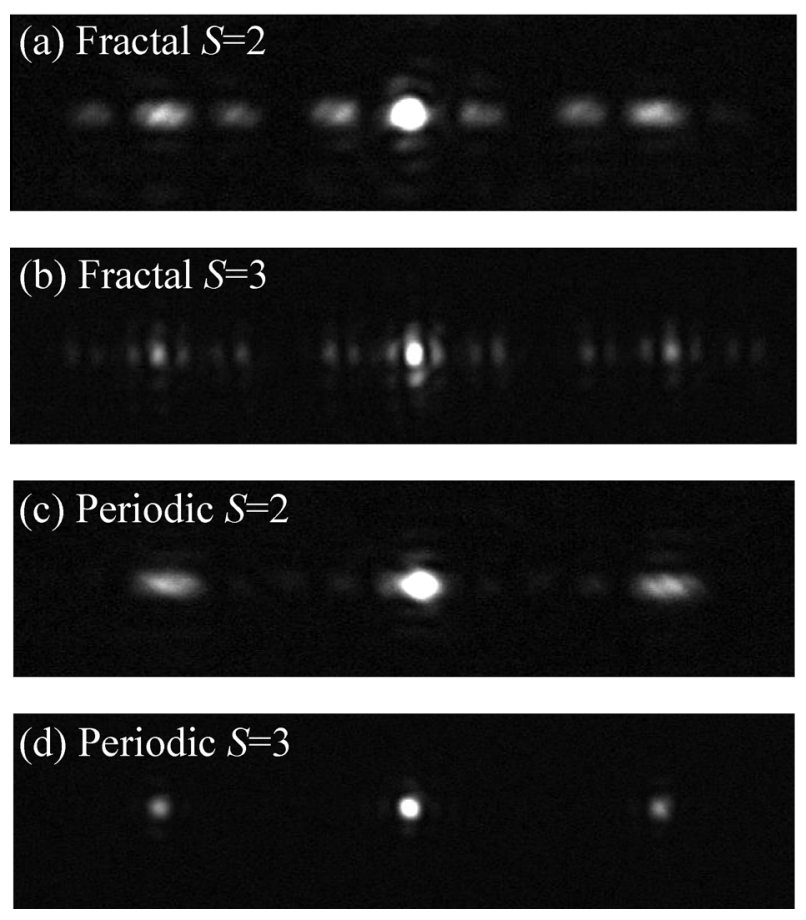

Figure 5. Experimental Fraunhofer diffraction patterns obtained with FGs of orders $S=2$ and $S=3$ and the corresponding periodic structures.

Focusing our attention only in the horizontal coordinate along the central axe $j=j_{0}$, the experimental data $I_{\exp }\left(i, j_{0}\right)$ are fit to the theoretical function $I_{t h}(i, N, \gamma)=N \cdot I_{\mathrm{F}}\left(\frac{i-i_{0}}{\gamma}, 0\right)$, where $N$ is a normalization factor and $\gamma$ is the scaling factor that transforms the horizontal coordinate $i$, defined in pixels, to frequencies $u=\frac{i-i_{0}}{\gamma}$. The parameters and their errors have been obtained according to the standard procedure of minimization of the $\chi^{2}$ merit function, defined as [13]:

$$
\chi^{2}(N, \gamma)=\sum_{i=1}^{764} \frac{\left(I_{\exp }\left(i, j_{0}\right)-I_{t h}(i, N, \gamma)\right)^{2}}{\sigma_{i}^{2}}
$$

and by assigning an error of $\sigma=3$ to the irradiance values corresponding to a mean noise registered. The points $i$ where the registered irradiance is saturated have not been considered in the above summatory. 
The dashed curves in Fig. 6 represent the experimental data obtained for the FGs at stages $S=2$ and $S=3$. The fitted functions are shown in the same figure with solid curves for comparison. The values of the fitted parameters are given in Table I. A good agreement is obtained between the theoretical and experimental data, being the regression coefficient higher than 0.9 in both fractal cases. Similar regression coefficients are obtained with the equivalent periodic structures (not shown in Fig. 6).
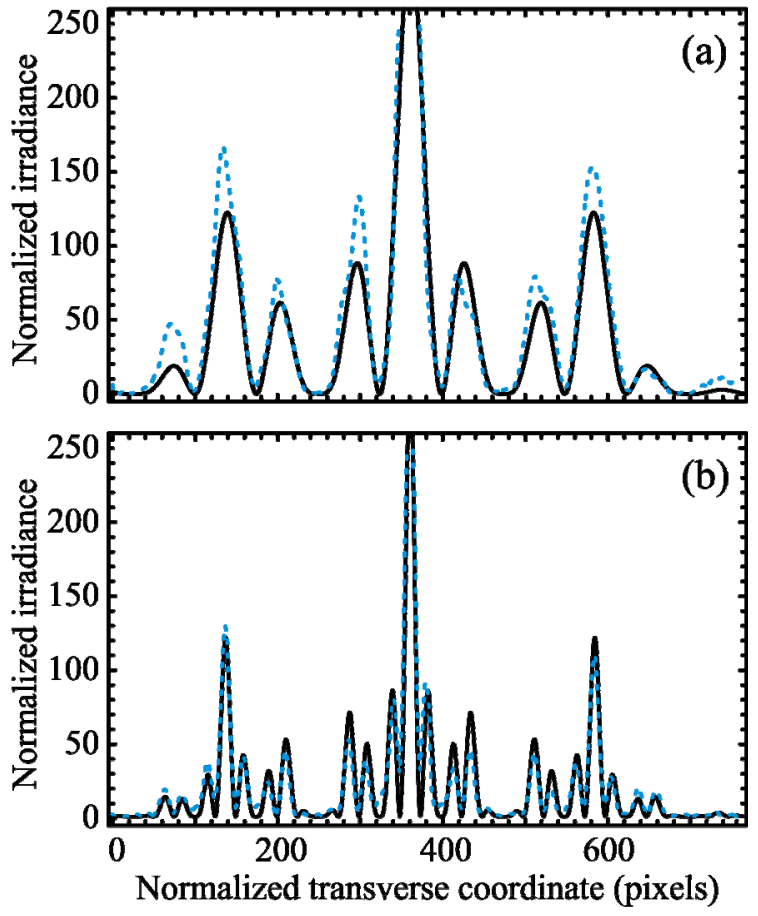

Figure 6. Experimental (dashed curve) and theoretical (solid curve) Fraunhofer diffraction patterns vs. the transverse coordinate in pixels for FGs at (a) $S=2$ and (b) $S=3$.

Table I. Experimental data fit.

FRACTAL $S=2$

\begin{tabular}{ccc}
\hline Mean position $\left(\boldsymbol{i}_{\mathbf{0}}, \boldsymbol{j}_{\mathbf{0}}\right)$ & $(361,281)$ pixels & $(362,274)$ pixels \\
Normalization Factor $\boldsymbol{N}$ & $354.1 \pm 0.6$ & $299.1 \pm 0.8$ \\
Scale Factor $\gamma$ & $(449.8 \pm 0.1)$ pixels & $(448.4 \pm 0.1)$ pixels \\
Regression coefficient & 0.92 & 0.91 \\
\hline
\end{tabular}




\section{Conclusions}

We have presented a simple optical method to analyze the Fraunhofer diffraction properties of one-dimensional gratings based on the Cantor set. With this method we have shown that optical processing can be performed using the standard equipment available in most undergraduate physics laboratories. In fact, the self-similarity of the diffraction patterns can be easily observed and recorded with this setup and measurements of the mathematical properties of the Cantor are also possible from experimental results. It is worth to be mentioned that with this method other one-dimensional or twodimensional fractals, such as polyadic Cantor sets, or other quasiperiodic structures, such as the Fibonacci gratings, can be studied. In our opinion this result could be a strong motivation for the students to go into the field of optical processing in depth.

\section{Acknowledgments}

We acknowledge the financial support from Grants DPI2008-02953 and TRA2009-0215, Ministerio de Ciencia e Innovación, Spain. We also acknowledge the support from Generalitat Valenciana (PROMETEO2009-077 and ACOMP/2010/052) and Universidad Politécnica de Valencia (PAID-05-09 and PAID-06-08). This work has been developed by Teaching Innovation Groups from the Universidad Politécnica de Valencia (e-MACAFI) and the Universitat de València (GCID35/2009).

\section{References}

[1] Mandelbrot B B 1982 The Fractal Geometry of Nature (Freeman, San Francisco, Calif.).

[2] Ficker T and Benesovký P 2002 Deterministic fractals Eur. J. Phys. 23403. 
[3] Sakurada Y, Uozumi J and Asakura T 1992 Fresnel diffraction by 1-D regular fractals Pure Appl. Opt. 129.

[4] Trabocci O, Granieri S and Furlan W D 2001 Optical propagation of fractal fields. Experimental analysis in a single display J. Mod. Optics 481247.

[5] Uozumi J, Peiponen K E, Savolainen M and Silvennoinen R 1994 Demostration of diffraction by fractals Am. J. Phys. 62283.

[6] Jaggard A D and Jaggard D L 1998 Cantor ring difractals Opt. Comm. 158141.

[7] Lehman M 2001 Fractal diffraction gratings built through rectangular domains Opt. Commun. 19511.

[8] Zunino L and Garavaglia M 2003 Fraunhofer diffraction by Cantot fractals with variable lacunarity J. Mod. Opt. 50717.

[9] Saavedra G, Furlan W D and Monsoriu J A 2003 Fractal zone plates Opt. Lett. 28 971.

[10] Monsoriu J A, Furlan W D, Saavedra G and Giménez F 2007 Devil's lenses Opt. Express 15, 13858.

[11] Essex C and Herenberg M A H 1990 Fractal dimension: Limit capacity or Husdorff dimension? Am. J. Phys. 58986.

[12] Goodman J W 1996 Introduction to Fourier optics (McGraw-Hill, New York).

[13] Bevington P and Robinson D K 1993 Data reduction and error analysis for the physical sciences (McGraw Hill, New York). 